\title{
"Striving for normalcy" was the core process in the symptom experience related to the threat of rejection after a lung transplant
}

DeVito Dabbs AD, Hoffman LA, Swigart V, et al. Striving for normalcy: symptoms and the threat of rejection after lung transplantation. Soc Sci Med 2004;59:1473-84.

\section{$Q$ In lung transplant recipients, what is the psychosocial process of the symptom experience associated with the threat of organ rejection?}

\section{DESIGN}

Grounded theory.

\section{SETTING}

University based pulmonary transplant centre.

\section{PARTICIPANTS}

14 lung transplant recipients who were 28-69 years of age (mean age 54 y, 50\% men, $93 \%$ white). Mean time since transplantation was 2.3 years (range $27 \mathrm{~d}$ to $9 \mathrm{y}$ ). 6 participants had $>3$ rejection episodes, 7 had $1-2$ episodes, and 1 had no episode.

\section{METHODS}

Participants were recruited using purposive and theoretical sampling methods to ensure variability in age, sex, underlying lung disease, previous rejection, and time since transplantation. Audiotaped, face to face interviews lasting 45-60 minutes and using open ended questions were done. Data collection, analysis, comparison, verification, and theoretical explanation were done concurrently. Interview questions were modified to compare and verify emergent concepts. 24 weeks after the initial interview, participants were contacted by telephone to verify findings. A substantive theory on the symptom experience associated with the threat of rejection was constructed. Use of various data sources and participant input about convergent and divergent experiences ensured the trustworthiness of findings.

\section{MAIN FINDINGS}

The core process identified with the symptom experience associated with the threat of rejection after lung transplantation was striving for normalcy. This was a dynamic psychosocial process involving 4 stages: naïveté, vulnerability, discovery, and insight. Progression through the stages was not necessarily linear, sequential, or predictable in duration. The elements common to each of these stages were initiating events, symptom response, and dialectics (dichotomous labels used to depict the simultaneous, interconnected, yet polar realities held by participants at each stage of the process); each stage was labelled with a corresponding descriptor of the aspect of striving for normalcy.

In the naivveté stage, participants were expecting normalcy. The initiating event was the normalisation of numbers, which referred to normal oxygen levels, spirometry, and pulmonary function tests. Participants' symptom responses reflected typical laymen's responses. The dialectic was elation versus realism: tension existed between the elation of participants as they began to feel normal again and knowledge that rejection was a real threat.

In the vulnerability stage, participants felt a jeopardising normalcy when their naïve expectation of normalcy was replaced by surprise and disappointment at the onset of an episode of acute rejection. The way in which participants responded to their symptoms reflected their newly admitted vulnerability and admission of the reality for complications of the transplant. The dialectic was nonchalance versus vigilance, specifically the tension between nonchalant feelings of participants who For correspondence: Dr A DeVito Dabbs, School of Nursing, University of Pittsburgh, Pittsburgh, PA, USA. ajdst42@pitt.edu

Sources of funding: National Institute of Nursing Research; Pennsylvania Nurse Foundation; Pauline Thompson Research Award; Enid Goldberg Research Award; and Sigma Theta Tau, Eta Chapter. had not yet experienced rejection and the vigilance that the transplant team expected.

The discovery stage involved recognising disparities from normalcy. Participants discovered that symptoms were ambiguous, and that often, symptoms of rejection were similar to those associated with common illnesses (eg, cold, flu, or pulmonary infection). When participants became aware of symptoms, they searched for the cause of the symptoms and considered whether the cause might be transplant rejection. The dialectic was certainty versus ambiguity. Tension existed between participants' desired certainty about the cause of their symptoms and their ability to tolerate the ambiguity of rejection symptoms.

The insight stage comprised doing your part for normalcy in illness. Participants recognised their role in achieving as normal a life as possible. Their symptom behaviours were proactive rather than reactionary. The dialectic was independence versus interdependence: tension existed between participants' desired independence as they strived for normalcy and the need for interdependence with the transplant team.

\section{CONCLUSIONS}

In lung transplant recipients, the core process of the symptom experience associated with the threat of rejection was striving for normalcy. This process linked the 4 stages of symptom experience and interpretation: naiveté, vulnerability, discovery, and insight. Each stage was marked by an initiating event, symptom response, and dialectics.
Commentary

he study by DeVito Dabbs et al builds on the current body of knowledge of the experiences of organ transplant recipients and the struggle to optimise quality of life. The threat of rejection is the critical obstacle in returning to normalcy, and the way in which an individual processes the threat and uses knowledge and symptoms to contain the threat is the concept of interest in this study. Lung transplant recipients offered unique insight into the complex psychosocial issues surrounding life after transplantation. Although previous descriptive studies have shown that these people struggle with symptom management and adaptation to a new transplanted organ, ${ }^{1}$ DeVito Dabbs et al used a grounded theory approach to develop an emergent theory of the experience of striving for normalcy. Each stage of data collection and verification were well described, and care was taken to validate findings with study participants. Study limitations include a lack of cultural diversity and centre specificity; thus, further verification of the constructs of this new theory is warranted.

The theory of "striving for normalcy" lays the groundwork for additional theory testing in lung transplant recipients and others who strive to improve the lives of organ transplant recipients. Nurses who deal with the day to day care of lung transplant recipients now have an emerging model to help guide and test interventions to assist patients in attaining a sense of normalcy.

Rebecca P Winsett, RN, PhD

University of Tennessee Health Science Center, Memphis, Tennessee and The University of Southern Indiana, Evansville, Indiana, USA

Dew MA, Switzer GE, Goycoolea JM, et al. Does transplantation produce quality of life benefits? A quantitative analysis of the literature. Transplantation 1997; 64:1261-73. 